\title{
Variability of food and nutrient composition measured through adherence to the Mediterranean diet in Mediterranean and non-Mediterranean regions
}

\author{
V.E. Awuzudike ${ }^{1}$, A. Jennings ${ }^{2}$, J.V. Craig ${ }^{3}$ and A.A. Welch ${ }^{3}$ \\ ${ }^{1}$ School of Biological Sciences, \\ ${ }^{2}$ Department of Nutrition and \\ ${ }^{3}$ Department of Public Health \& Primary Care, Norwich Medical School, University of East Anglia NR4 7TJ, UK
}

A Mediterranean style dietary pattern has been linked to several beneficial health outcomes in the Mediterranean regions ${ }^{(1)}$. These outcomes are achieved through the protective effects of the dietary components of the Mediterranean diet (MD) which contains nutrients with antioxidant and anti-inflammatory properties. Whilst a wealth of information exists focusing on the health benefits of the MD within the Mediterranean region, the benefits in non-Mediterranean regions have been less well established ${ }^{(2)}$. As the quantity and types of foods that contribute to the Mediterranean dietary pattern vary throughout Europe, and adherence to this pattern is calculated using distribution of foods within countries, this can impact on resultant food and nutrient intakes ${ }^{(3)}$. This study aimed to investigate if food and nutrient intakes within populations that reported MD adherence differed across countries, methods of classifying the MD, and dietary assessment methods, as a means of comparing the variability in food and nutrient composition of the MD.

Research was conducted using the EMBASE database. Seventy-two systematic reviews were found using the MeSH term "Mediterranean diet". Systematic reviews were subsequently reviewed to check if they fit the study inclusion criteria which 1) focused on the MD in observational studies 2) populations that did not include children, lactating or pregnant women 3) reported MD adherence according to mean/median dietary intake 4) had a clear focus on a health outcome and 5) defined the MD a-priori. Currently, eighteen systematic reviews have been assessed with 15 being rejected. Three reviews gave rise to nine primary papers which complied with the inclusion criteria. A total of eighteen primary papers have been found with the remaining nine found through initial scoping literature searches. Studies that reported dietary intakes in servings and/or by week were converted into g/day using relevant conversion calculations based on estimates of food portion values. The values for foods and nutrients according to the highest and lowest category of adherence to the MD were recorded for each study and the difference between both values was calculated. Data for intakes of fruits and vegetables and nutrient intakes are reported here. The means and standard deviation of the differences were calculated using Microsoft Excel.

Of the eighteen papers found, fourteen (78\%) reported data on MD adherence by food groups. Within these, eleven reported fruit and vegetables as separate categories. According to the classifications of the MD, across countries, the highest intakes of vegetables ranged from 1875-171 g/day and the lowest from 1245-78 g/day, with differences between high and low adherence ranging from 630-51 g/day. Fruit intakes ranged from 935-129 g/day and 488-53 g/day respectively, with differences of 535-62 g/day. The mean difference in vegetable intake was $198 \mathrm{~g}$ /day $(\mathrm{SD}=159)$ and coefficient of variation $(\mathrm{CV}) 80 \%$. In fruits the mean difference was $187 \mathrm{~g}$ /day $(\mathrm{SD}=138)$ and $\mathrm{CV}=74 \%$. Fifteen studies $(83.3 \%)$ reported details of adherence for macronutrients and only five $(27 \%)$ details for micronutrients.

These results highlight the vast variability in fruit and vegetable intake, and reported nutrient composition, measured through the MD classification across Mediterranean and non-Mediterranean countries. This is an ongoing study with continued searches ongoing for papers reporting food and nutrient adherence to the Mediterranean diet.

1. Misirli G, Benetou V, Lagiou P et al. (2012) Am J Epidemiol 176 1185-1192.

2. Trichopoulou A, Bamia C, Trichopoulou D (2009) BMJ 339 26-29.

3. Slimani A, Fahey M, Welch A et al. (2002) Public Health Nutr 5 1311-1328. 